\title{
Predicting Extubation Outcome by Cough Peak Flow Measured Using a Built-in Ventilator Flow Meter
}

\author{
Florent Gobert MD, Hodane Yonis MD, Romain Tapponnier MD, Raul Fernandez, \\ Marie-Aude Labaune, Jean-François Burle, Jack Barbier, Bernard Vincent, Maria Cleyet, \\ Jean-Christophe Richard MD PhD, and Claude Guérin MD PhD
}

\begin{abstract}
BACKGROUND: Successful weaning from mechanical ventilation depends on the patient's ability to cough efficiently. Cough peak flow (CPF) could predict extubation success using a dedicated flow meter but required patient disconnection. We aimed to predict extubation outcome using an overall model, including cough performance assessed by a ventilator flow meter. METHODS: This was a prospective observational study conducted from November 2014 to October 2015. Before and after a spontaneous breathing trial, subjects were encouraged to cough as strongly as possible before freezing the ventilator screen to assess CPF and tidal volume $\left(V_{T}\right)$ in the preceding inspiration. Early extubation success rate was defined as the proportion of subjects not re-intubated $48 \mathrm{~h}$ after extubation. Diagnostic performance of $\mathrm{CPF}$ and $\mathrm{V}_{\mathrm{T}}$ was assessed by using the area under the curve of the receiver operating characteristic curve. Cut-off values for $C P F$ and $V_{T}$ were defined according to median values and used to describe the performance of a predictive test combining them with risk factors of early extubation failure. RESULTS: Among 673 subjects admitted, 92 had a cough assessment before extubation. For the 81 subjects with early extubation success, the median CPF was $-67.7 \mathrm{~L} / \mathrm{min}$, and median $V_{T}$ was $0.646 \mathrm{~L}$. For the 11 subjects with early extubation failure, the median CPF was $-57.3 \mathrm{~L} / \mathrm{min}$, and median $\mathrm{V}_{\mathrm{T}}$ was $0.448 \mathrm{~L}$. Area under the curve was $0.61(95 \% \mathrm{CI}$ 0.37-0.83) for CPF and $0.64(95 \%$ CI $0.42-0.84)$ for $\mathrm{CPF} / \mathrm{V}_{\mathrm{T}}$ combined. After dichotomization $\left(\mathrm{CPF}<-60 \mathrm{~L} / \mathrm{min}\right.$ or $\left.\mathrm{V}_{\mathrm{T}}>0.55 \mathrm{~L}\right)$, there was a synergistic effect to predict early extubation success $(P<.001)$. The predictive value of success reached $94.2 \%$ for $\mathrm{CPF} / \mathrm{V}_{\mathrm{T}}$ combined. The overall model including $\mathrm{pH}$ before extubation $<7.45$ reached a $66.7 \%$ predictive value of failure. CONCLUSIONS: CPF measured using the flow meter of an ICU ventilator was able to predict extubation success and to build a composite score to predict extubation failure. The results were close to that found in previous studies that used a dedicated flow meter. This could help to identify high-risk subjects to prevent extubation failure. (ClinicalTrials.gov registration NCT02847221.) Key words: cough; weaning; extubation; critical care; respiratory failure; prognosis. [Respir Care 2017;62(12):1505-1519. (c) 2017 Daedalus Enterprises]
\end{abstract}

\section{Introduction}

Extubation should commonly follow a successful spontaneous breathing trial (SBT) in patients mechanically ven-

\footnotetext{
The authors are affiliated with the Hospices Civils de Lyon, Hôpital de la Croix Rousse, Service de Réanimation Médicale, Lyon, France. Drs Gobert, Yonis, Richard, and Guérin are also affiliated with the Université de Lyon, Université Claude Bernard Lyon 1, Villeurbanne, France. Dr Guérin is also affiliated with INSERM 955 Equipe 13, Créteil, France.
}

The authors have disclosed no conflicts of interest. tilated in the ICU. The success of extubation can then be affected by several factors, such as cardiogenic pulmonary edema, ${ }^{1}$ laryngeal edema, or new acute respiratory distress requiring re-intubation. ${ }^{2}$ Furthermore, when the quantity

\footnotetext{
Correspondence: Florent Gobert MD, Centre Hospitalier Universitaire de Lyon, Hôpital Neurologique et Neurochirurgical Pierre Wertheimer, 59 Boulevard Pinel, 69500 Bron Cedex, France. E-mail: gobert.flo@gmail.com.
}

DOI: $10.4187 /$ respcare .05460 
of respiratory secretions is too great for the spontaneous cough to expel, this may lead to extubation failure, which is reported to occur in almost $89 \%$ of re-intubation cases, whereas only $39 \%$ of subjects with extubation success

\section{See the Related Editorial on Page 1611}

have abundant secretions. ${ }^{3}$ This has important clinical implications because re-intubation after extubation failure is associated with clinical status worsening (as assessed by the increase in daily Sequential Organ Failure Assessment scores) $)^{4}$ and has been suggested to be associated with a higher ICU mortality rate (between 25 and 50\%). ${ }^{5}$ To reach a high early extubation success rate (within 48 or $72 \mathrm{~h}$, depending on the authors), verifying the patient's ability to protect the airways before endotracheal tube (ETT) removal is recommended. ${ }^{6}$ But the lack of practical and wellestablished recommendations is likely to explain the heterogeneous reported extubation failure rate (from 14 to 23\%). ${ }^{7}$

Rationalizing cough assessment could be one way to improve medical practice, because it would allow the identification of the subgroup of patients with an increased risk of extubation failure. Based on objective cough measurement, one could propose individualized protocols of airway management to avoid re-intubation. ${ }^{8}$ Cough strength can, for example, be evaluated by cough peak flow (CPF). A CPF cut-off value of $-60 \mathrm{~L} / \mathrm{min}$ for voluntary and unassisted cough in a medical ICU population has found some consensus, ${ }^{8-11}$ but its accuracy is questionable because the pre-cough biomechanical thoracic features are not taken into account. Furthermore, the specificity of CPF assessment for extubation prediction is not known, because one study that reported the prognostic value of $\mathrm{CPF}$ did not use a standardized SBT, 12 and therefore it was not possible here to delineate the prediction of successful weaning from mechanical ventilation rather than cough per se (because the glottis cannot be entirely closed in the presence of an $\mathrm{ETT}^{13}$ ). In this case, CPF would reflect the general performance of the respiratory system.

More generally, CPF is not currently used routinely for the assessment of extubation ability for several other reasons. For example, it has not been demonstrated that CPF assessment can be performed by non-specialized caregivers, and furthermore this requires specific devices. ${ }^{8,9}$ The use of a dedicated flow meter has technical limitations for routine use because it is not available in all ICUs and it requires connection to a bacterial filter to avoid cross-contamination. Because generalization is limited, CPF failed to modify routine care. ${ }^{3,8}$ In parallel, alternative methods that aimed to formalize assessment were developed, such as the semi-quantitative cough strength score (from 0 to 5), the magnitude of endotracheal secretions (none, mild, moderate, or abundant), and the white-card test (presence of secretions propelled onto the card held $1-2 \mathrm{~cm}$ from the ETT).

\section{QUICK LOOK}

\section{Current knowledge}

After a successful spontaneous breathing trial, assessing cough ability is a classic prerequisite before the medical decision of extubation. Subjective evaluations of cough effort are currently used in daily clinical practice, but previous studies have proposed objective cough peak flow thresholds using a dedicated flow meter requiring the patient's disconnection from the ventilator.

\section{What this paper contributes to our knowledge}

The use of a built-in flow meter to assess cough peak flow was validated. A previously proposed threshold $(-60 \mathrm{~L} / \mathrm{min})$ was confirmed as having a high positive predictive value for extubation success. The prediction of extubation failure was increased by a composite score, including the $\mathrm{V}_{\mathrm{T}}$ in the preceding inspiration and the $\mathrm{pH}$ before extubation.

The objective of the present study was to predict extubation outcome in ICU subjects by assessing cough performance before and after SBT in addition to other clinical and biological parameters available at the time of extubation. Furthermore, the ability of CPF to predict other end points, including overall extubation outcome, and mortality was evaluated.

\section{Methods}

\section{Bench Study Validating the Use of the Built-in Ventilator Flow Meter}

The experimental design is described in Figure 1A CoughAssist device (Philips Respironics, Murrysville, Pennsylvania) was used to inflate a pneumatic lung model (TTL 5601 i, Michigan Instruments, Grand Rapids, Michigan) over a range of nominal pressures $(10,20,30$, 40 , and $50 \mathrm{~cm} \mathrm{H}_{2} \mathrm{O}$ ). Lung model compliance was set to $30 \mathrm{~mL} / \mathrm{cm} \mathrm{H}_{2} \mathrm{O}$, and airway resistance was set to $5 \mathrm{~cm}$ $\mathrm{H}_{2} \mathrm{O} / \mathrm{L} / \mathrm{s}$. Flow was measured using a linear pneumotachograph (TSD 137 G, Hans Rudolph, Kansas City, Kansas). Airway pressure and flow were measured using a Biopac M150 data logger and Acqknowledge software 4.4.2 (BIOPAC Systems, Goleta, California).

Four sizes of ETTs were tested (7.0-, 7.5-, 8.0-, and 8.5-mm internal diameter). For each ETT and inflation pressure, 2 kinds of passive exhalation to atmosphere were investigated: through ETT alone (scenario 1 in Fig. 1) and through ETT, expiratory line, and expiratory valve of the EvitaXL ventilator (Dräger, Lübeck, Germany; scenario 2 in Fig. 1). 
A

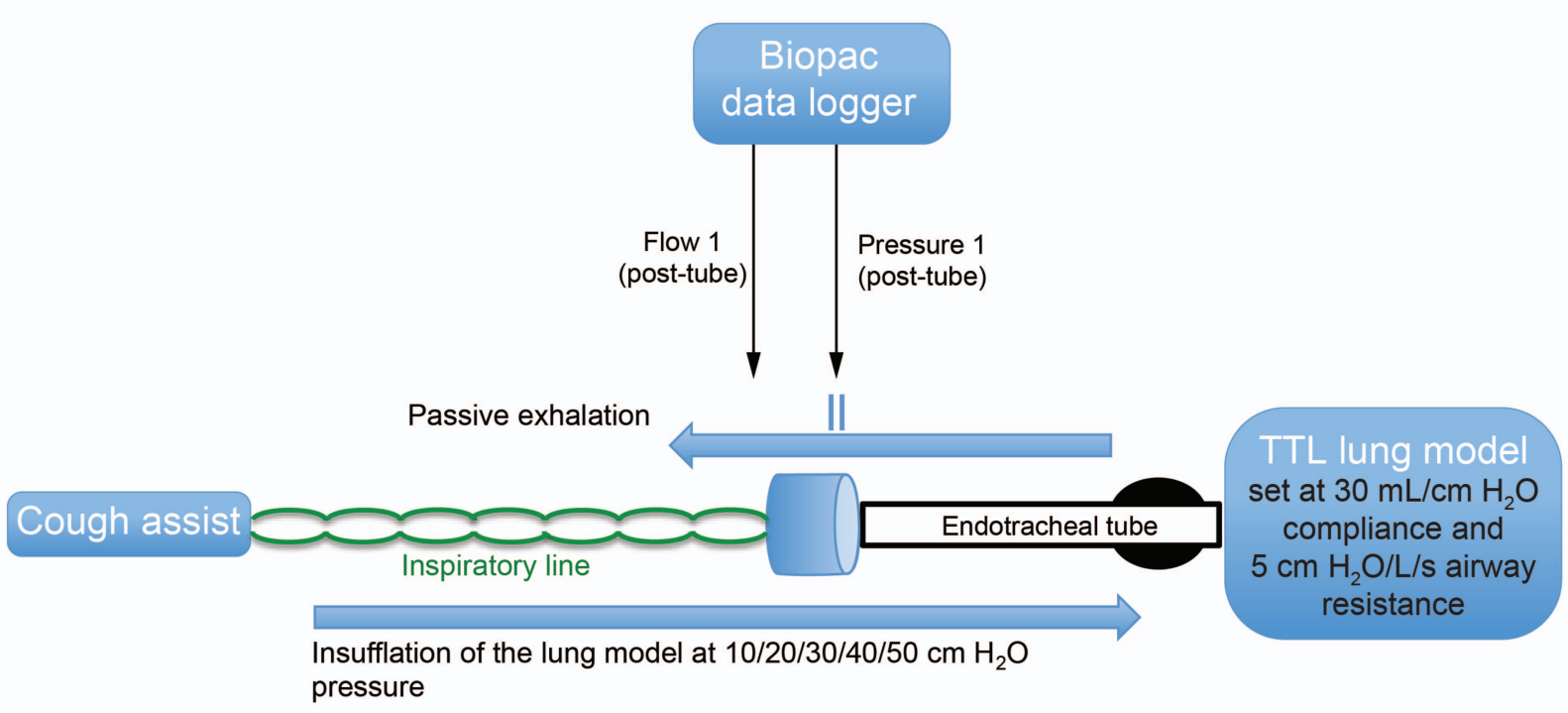

B Passive expiration through the expiratory circuit

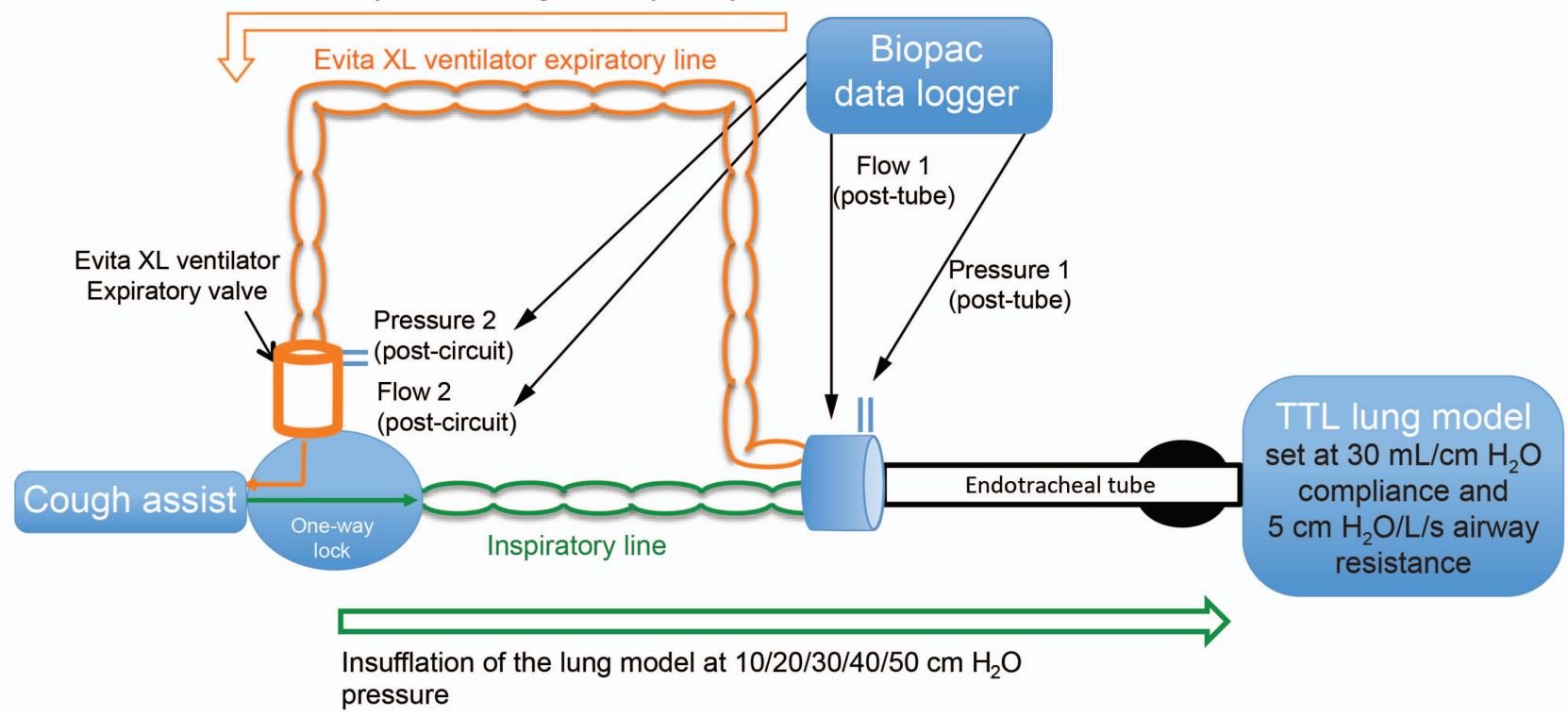

Fig. 1. Experimental setup. A: After increasing levels of insufflation pressure in the lung model, passive expiration from the lung model to atmospheric pressure (through endotracheal tube alone) is recorded 3 times for both pressure and flow assessments. B: After the same increasing levels of insufflation pressure in the lung model, passive expiration is recorded 3 times (for both pressure and flow assessments) from the lung model through the endotracheal tube and the expiratory circuit (line and valve).

For each condition, 3 consecutive breaths were delivered, and maximal inspiratory pressure and peak expiratory flow were measured and analyzed. Peak expiratory flow was measured downstream of the ETT for scenario 1 and also downstream of the ETT expiratory valve for scenario 2 .

\section{Clinical Study: Population Selection}

A 1-y prospective observational study was performed in a 15-bed medical ICU of the University Hospital in Lyon,
France, from November 10, 2014, to October 30, 2015. Inclusion criteria were: age $>18 \mathrm{y}$, intubation $>24 \mathrm{~h}$, absence of decision to withdraw life-supporting care, eligibility for scheduled SBT and then extubation trial in the ICU (excluding extubation in the theater for high-risk extubation in case of difficult intubation and cervical tumors; or subjects transferred to another ICU while intubated), mechanical ventilation from an EvitaXL ventilator using an orotracheal tube (no tracheostomy), neurological status compatible with cough on demand (Glasgow coma score $>8$; namely eye response $\geq 1$, motor response $=6$, ver- 

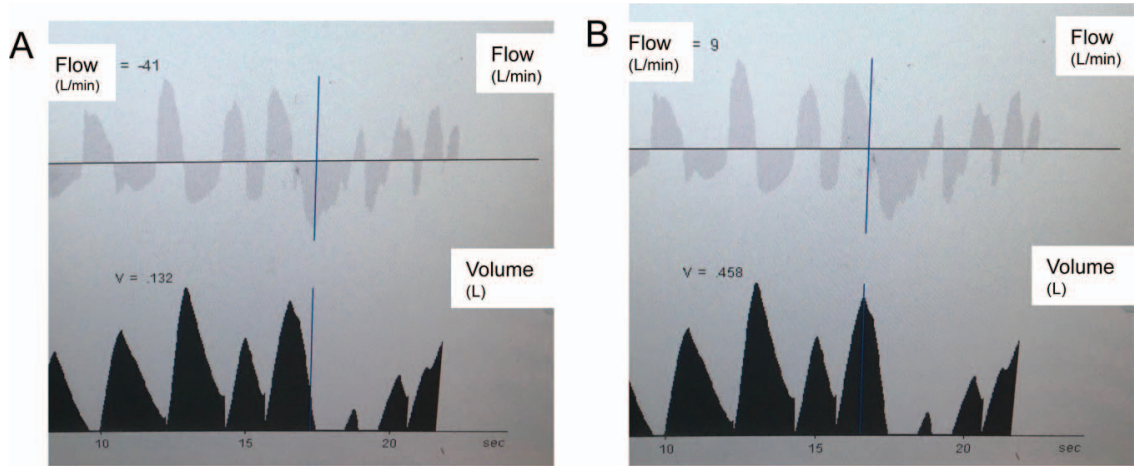

Fig. 2. Ventilator screenshots. After a cough effort, the screen is frozen and the cursor is scrolled up to the value to be recorded. Cough peak flow $(C P F)$ is shown in gray $(A)$, and $V_{T}$ is shown in black $(B)$.

bal response $\geq 1$ ), and confirmation of the subject's agreement to participate. Non-scheduled extubation, patient refusal, and legally incompetent adults were non-inclusion criteria. The protocol was approved by the local ethics committee on June 19, 2014 (institutional review board approval 11263), and informed consent was waived.

\section{Study Protocol}

In this ICU, the SBT is routinely standardized as follows. ${ }^{14}$ Daily, between 6 and 8 AM, weaning criteria are checked, and when all are present, the ventilator is set to pressure support ventilation at predetermined settings (inspiratory pressure $=7 \mathrm{~cm} \mathrm{H}_{2} \mathrm{O}, \mathrm{PEEP}=4 \mathrm{~cm} \mathrm{H}_{2} \mathrm{O}$, inspiratory trigger $=2 \mathrm{~L} / \mathrm{min}$, expiratory cycling $=25 \%$ peak flow, $\mathrm{F}_{\mathrm{IO}_{2}}=0.40$, with a heated humidifier). These settings are planned to be applied for $1 \mathrm{~h}$ (if no chronic respiratory failure), $2 \mathrm{~h}$ (in the case of chronic respiratory failure or COPD), or at least $12 \mathrm{~h}$ (in the case of neuromuscular disease). Some SBTs are prolonged if patients have not fulfilled the clinical criteria for extubation but do not require a higher pressure support. During this period, predetermined criteria are checked every $20 \mathrm{~min}$ and are used to define SBT outcome. SBT success is defined as all of the following criteria being present: breathing frequency $<35 / \mathrm{min}$, heart rate variation $<20 \%$ from preSBT, systolic arterial pressure variation $<20 \%$ from pre-SBT, and no acute respiratory distress (agitation, perspiration, paradoxical thoracic movements during breathing). Occurrence of any of these within the time frame described above defines SBT failure, leading to the resumption of ventilator support before the SBT. ${ }^{15}$ Medical extubation decisions should be based on arterial blood gas results.

CPF was assessed before and after SBT with the built-in flow meter (hot wire technology, Spirlog, EvitaXL, Dräger; flow resolution $=0.1 \mathrm{~L} / \mathrm{min}$; temporal resolution $=25 \mathrm{~Hz}$; precision $\pm 8 \%$ ) incorporated into the ICU ventilator without disconnection from the ventilator. The procedure was performed as follows. The subject was placed in a semi-recumbent position, and the clinician in charge explained to him/her the goals and steps of the study. The caregiver in charge (a physician or a physiotherapist for early [before SBT] and late [before extubation] assessments; a nurse for early assessments only) encouraged the subject to cough as strongly as possible after having attempted to reach total lung capacity in synchronization with the mechanical breath. The subject was instructed to perform 3 consecutive cough efforts. After each cough effort, the ventilator screen was frozen, and the cursor was scrolled to record the maximal value of CPF for each cough effort (Fig. 2). Tidal volume $\left(\mathrm{V}_{\mathrm{T}}\right)^{16}$ in each preceding inspiration was also measured and regarded as an assessment of the lung volume at the end of the inspiratory effort. The assessment was scheduled to be performed at 2 time points, immediately after SBT onset and before extubation, resulting in early $\mathrm{CPF}$, early $\mathrm{V}_{\mathrm{T}}$, late $\mathrm{CPF}$, and late $\mathrm{V}_{\mathrm{T}}$. The results of the 3 measurements were given to one of the authors (FG), who stored these in a file that was not accessible to caregivers (limiting the influence of the results on the primary end point through bias). Routine care was applied during the $48 \mathrm{~h}$ after extubation, with systematic use of noninvasive mechanical ventilation restricted to hypercapnic COPD,${ }^{17}$ hypercapnia after SBT $\left(\mathrm{P}_{\mathrm{aCO}_{2}}>45 \mathrm{~mm} \mathrm{Hg}\right)$, or other selected indications. ${ }^{14}$ In the case of secondary acute respiratory failure, re-intubation was rapidly considered with respect to the literature. $^{2}$

\section{Clinical and Biological Data Collected}

Demographic features, cause of intubation, Simplified Acute Physiology Score II, and Charlson comorbidity index at the time of ICU admission, Sequential Organ Failure Assessment score, number of days under invasive/noninvasive/total mechanical ventilation, weaning features (number of previous SBTs, duration of the last SBT, arterial blood gas before extubation), duration of mechan- 


\section{Predicting Extubation With Ventilator-Measured CPF}

ical cough assistance after extubation, and ICU/hospital admission time were prospectively recorded.

\section{Definition of End Points}

The primary end point of the predictive model was early extubation outcome. The early extubation success rate was defined as the proportion of subjects who were alive and not re-intubated $48 \mathrm{~h}$ after the scheduled extubation, as usually proposed in large randomized controlled trials. ${ }^{2}$

The secondary end points were the SBT success rate, as defined previously from the absence of intolerance criteria during SBT; the overall extubation success rate, defined as the proportion of subjects who were alive and not reintubated at any time after scheduled extubation; and the rate of ICU and hospital mortality.

\section{Assessment of the Performance of CPF and $V_{T}$ to Predict Outcomes}

The mean of the $3 \mathrm{CPF}$ and $\mathrm{V}_{\mathrm{T}}$ assessment values for each subject at each time point was calculated. A subject could not be included twice in the study to avoid repeated data. Late CPF and late $\mathrm{V}_{\mathrm{T}}$ were used to predict early extubation success, overall extubation success, and ICU and hospital mortality. Early CPF and early $\mathrm{V}_{\mathrm{T}}$ were used to predict SBT outcome.

\section{Step-by-Step Building of a General Model for Extubation Outcome Prediction}

For extubation outcome prediction, $\mathrm{V}_{\mathrm{T}}$ was used in addition to CPF. The rationale for this strategy is as follows. The strength of cough depends on different factors, one of them being the size of $\mathrm{V}_{\mathrm{T}}$ before the cough effort; the higher the $\mathrm{V}_{\mathrm{T}}$ pre-cough, the higher the CPF should be if all other factors are comparable. Concerning the operating volume, 2 levels can be reached, either the functional residual capacity or the total lung capacity. ${ }^{18}$ The former is more reproducible because the cough effort starts at the end of normal expiration but may be difficult to perform at the bedside in ICU patients with a possible neurological impairment. The latter is more natural for patients because they usually breathe deeply before cough to ensure a better cough performance, which is a better indicator of patients' ability to prevent excess respiratory secretions after extubation. We therefore first instructed the subject to try to reach total lung capacity and then to cough, and we recorded the $\mathrm{V}_{\mathrm{T}}$ before cough as a supplementary measure, demonstrated to be linearly correlated with the cough expiratory volume in healthy subjects. ${ }^{19}$

A composite cough strength score was defined using $\mathrm{CPF}$ and $\mathrm{V}_{\mathrm{T}}$ that were dichotomized according to the median values of the early extubation success/early extuba- tion failure groups reflecting high/low cough ability. For CPF only, the accordance of the threshold with the literature was evaluated post hoc (no comparison available in literature for $\mathrm{V}_{\mathrm{T}}$ ).

We then analyzed specifically the group of subjects with a composite cough strength score associating both low $\mathrm{CPF}$ and low $\mathrm{V}_{\mathrm{T}}$ to further refine extubation prognosis, by analyzing clinical and biological parameters. For this, parametric or non-parametric tests (after the Shapiro-Wilk normality test) were used in accordance with the technique proposed for the overall population description (see the Statistical Analysis section). After having defined convenient thresholds for these selected variables, the extubation prediction score was then defined as a multimodal criterion to refine the extubation prognosis.

\section{Statistical Analysis}

For the bench study, inspiratory pressures and peak expiratory flow were compared using a non-paired Student's $t$ test between scenario 1 and 2. For scenario 2, peak expiratory flows at the 2 locations were compared by using a paired $t$ test. The normal distribution of these variables was confirmed by the Shapiro-Wilk normality test.

For the clinical study, values are expressed as median of the mean values of CPF and $\mathrm{V}_{\mathrm{T}}$ (with associated interquartile range) and counts (percent per group). The coefficient of variation of $\mathrm{CPF}$ and $\mathrm{V}_{\mathrm{T}}$ was computed as the ratio of the SD to mean value over the 3 measurements in each subject. The difference between each of the 3 measurements was tested by a mixed linear model. The normal distribution of continuous variables was assessed using the Shapiro-Wilk normality test.

Median values were compared using non-parametric (Wilcoxon-Mann-Whitney test) or parametric (Student $t$ test) tests between non-paired groups ${ }^{9}$ at 2 phases of analysis: total population description and characterization of the parameters associated with early extubation outcome among subjects selected to have an increased risk according to cough performance assessed by the composite cough strength score.

Qualitative nominal variables (after dichotomization) were compared using the Pearson chi-square test for the performance of early $\mathrm{CPF} /$ early $\mathrm{V}_{\mathrm{T}}$, late $\mathrm{CPF} /$ late $\mathrm{V}_{\mathrm{T}}$, composite cough strength score, and extubation prediction score. Sensitivity, specificity, positive predictive value, negative predictive value, and the area under the curve of the receiver operating characteristic (ROC) curve together with $95 \%$ CI were assessed. For combined criteria, we first performed a logistic regression on both parameters and then applied the ROC method to the final general linear model. We used thresholds selected from the median values of CPF and $\mathrm{V}_{\mathrm{T}}$ in the present cohort to define the high or low values of both parameters. Correlation between 
Predicting Extubation With Ventilator-Measured CPF
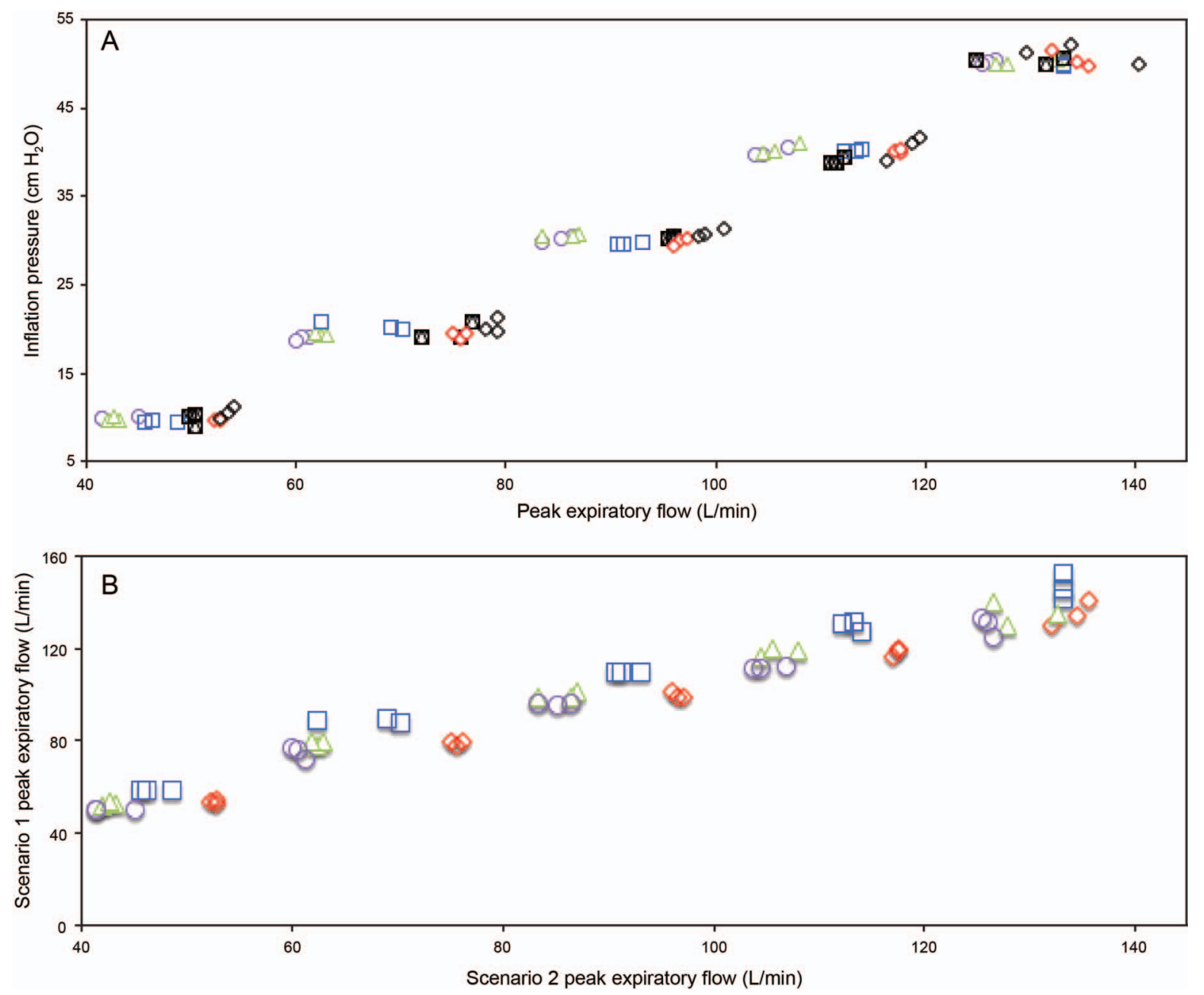

Fig. 3. A: Relationships of inspiratory pressure to flow for endotracheal tube of 7.0 (circles), 7.5 (triangles), 8.0 (squares), and 8.5 (diamonds) $\mathrm{mm}$ internal diameter when passive expiration happens through the endotracheal tube alone (colored symbols, purple, green, blue, and red, respectively; scenario 1 of the bench study, see Fig. 1) or through the combination of endotracheal tube and expiratory circuit of the EvitaXL ICU ventilator (black symbols; scenario 2 of the bench study, see Fig. 1). B: Relationships between expiratory flows pertaining to scenarios 1 and 2 of the bench study.

each parameter were analyzed by Pearson product-moment correlation.

Statistical analysis was performed using the Rcmdr package (RCore Team, 2015, R: A language and environment for statistical computing, R Foundation for Statistical Computing, Vienna, Austria). $P<.05$ was considered as significant.

\section{Results}

\section{Bench Study}

The bench study demonstrated a coherent increase of expiratory flow in function of inflation pressure (Fig. 3A). There was no statistically significant difference in peak expiratory flows between the 2 scenarios at each ETT size and inflation pressure tested (Fig. 3B).

\section{Characteristics of the Population}

During the study period, 673 patients were admitted to this ICU, of whom 319 were intubated and 109 were included (Fig. 4). The main reasons for non-inclusion ( $n=199 ; 62.4 \%$ of the intubated subjects) were: death before extubation $(n=73 ; 22.9 \%)$, intubation for $<24 \mathrm{~h}$ ( $n=30 ; 9.4 \%)$, use of another ventilator $(n=22 ; 6.9 \%)$, and autoextubation $(n=20 ; 6.3 \%)$. Among includable subjects, $\mathrm{CPF}$ and $\mathrm{V}_{\mathrm{T}}$ were not recorded for 46 subjects $(14.4 \%)$ at the time of early assessments and were not recorded for 28 subjects $(8.8 \%)$ at the time of late assessments.

Among the 92 subjects with late $\mathrm{CPF} /$ late $\mathrm{V}_{\mathrm{T}}$ recordings available, $81(88.0 \%)$ had early extubation success, and 71 (77.2\%) had overall extubation success. Sixteen subjects 


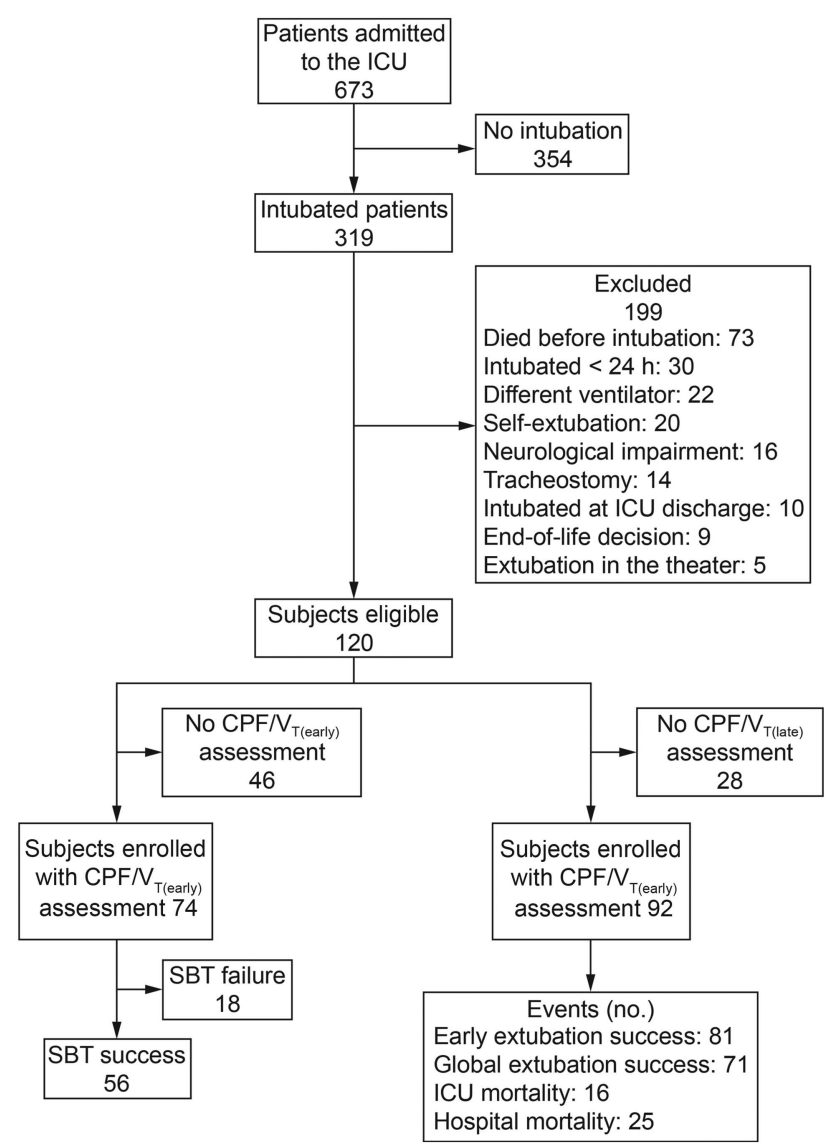

Fig. 4. Flow chart. CPF $=$ cough peak flow. SBT $=$ spontaneous breathing trial.

(17.4\%) died during their ICU stay, and 25 (27.2\%) died during their hospital stay. Among the 74 subjects with early CPF/early $\mathrm{V}_{\mathrm{T}}$ recordings, $56(75.7 \%)$ had SBT success.

Subjects with or without early extubation success are described in Table 1: Differences were statistically significant for the length of ICU stay, hospital and ICU mortality, and days of noninvasive ventilation (NIV) after early extubation failure. Among the reasons for early extubation failure, bronchial overload occurred in 3 cases (Table 2).

\section{CPF and $V_{T}$ Values in the Clinical Study}

Individual variations between assessments for late CPF and late $\mathrm{V}_{\mathrm{T}}$ according to extubation outcome are shown in Figure 5. For all late $\mathrm{CPF}$ and late $\mathrm{V}_{\mathrm{T}}$ values, the coefficient of variation of early CPF was 0.11 , and that of early $\mathrm{V}_{\mathrm{T}}$ was 0.20 ; for late CPF, it was 0.13 , and for late $\mathrm{V}_{\mathrm{T}}$ it was 0.21 . No statistically significant difference was observed between triplicate measures (late CPF: $P=.95$; late $\mathrm{V}_{\mathrm{T}}: P=.99$ ), confirming the use of mean values for predictive purposes. For the 92 subjects assessed for the primary end point, the median late $\mathrm{CPF}$ was -67.17 $\mathrm{L} / \mathrm{min}$, and median late $\mathrm{V}_{\mathrm{T}}$ was $0.640 \mathrm{~L}$.

Among subjects with early extubation failure, median (interquartile range) late CPF was $-67.7(-85.3$ to -57.3$) \mathrm{L} / \mathrm{min}$, and among those with early extubation success, this was -57.3 ( -84.5 to -42.8$) \mathrm{L} / \mathrm{min}$ (Fig. 6A and Table 3). Among those with early extubation failure, late $\mathrm{V}_{\mathrm{T}}$ was $0.646(0.477-0.892) \mathrm{L}$, and among those with early extubation success, it was $0.448(0.362-0.758) \mathrm{L}$ (Fig. 6B and Table 3).

The thresholds of late $\mathrm{CPF}$ and late $\mathrm{V}_{\mathrm{T}}$ selected for further analyses concerning early extubation success and to define the composite cough strength score were $-60 \mathrm{~L} / \mathrm{min}$ and $0.55 \mathrm{~L}$, respectively. Early extubation success was significantly associated with high late CPF $(P=.03$; Table 3$)$ but not with high late $\mathrm{V}_{\mathrm{T}}$ $(P=.08)$. The intrinsic performance for early extubation success prediction by late $\mathrm{CPF}$ alone was modest (sensitivity $=70.4 \%, 95 \%$ CI $60.4-80.3 \%$; specificity $=63.6 \%, 95 \%$ CI $35.2-92.1 \%$ ). The area under the curve of the ROC curve for late CPF was 0.61 (95\% CI $0.37-0.83)$, and that of late $\mathrm{V}_{\mathrm{T}}$ was 0.64 (95\% CI $0.42-$ 0.86; Table 3 and Fig. 7A).

\section{Performance of the Model Predicting the Primary End Point (Early Extubation Outcome)}

Early extubation success was better predicted using the composite cough strength score (Fig. 7B and Table 3): Late $\mathrm{CPF}<-60 \mathrm{~L} / \mathrm{min}$ or late $\mathrm{V}_{\mathrm{T}}>0.55 \mathrm{~L}$ was significantly associated with early extubation success $(P<.001)$, with a better intrinsic performance (sensitivity $=83.9 \%$, 95\% CI 75.9-91.9\%; specificity $=63.6 \%, 95 \%$ CI 35.2-92.1\%). The composite cough strength score had a high positive predictive value $(94.4 \%, 95 \%$ CI $89.5-99.4 \%)$ for early extubation success but a low negative predictive value $(35.0 \%, 95 \%$ CI $6.8-63.2 \%)$, indicating that weak cough performed poorly to predict early extubation failure. Composite cough strength score remained more accurate than late $\mathrm{CPF}$ alone (negative predictive value $=22.6 \%$, 95\% CI 0-47.3\%; Table 3), despite late CPF and late $\mathrm{V}_{\mathrm{T}}$ being statistically correlated $\left(\mathrm{R}^{2}=-0.56, P<.001\right)$. The area under the curve value for the ROC curve for the combined criteria (late $\mathrm{CPF}$ and late $\mathrm{V}_{\mathrm{T}}$ ) was $0.64(95 \% \mathrm{CI}$ 0.42-0.84).

Among those with an unfavorable composite cough strength score, the only significant parameter in the overall model was $\mathrm{pH}$ before extubation (normal distribution, mean value in the failure group $=7.40$; mean value in the success group $=7.45 ; P=.049$ ). This result was specific to this subgroup (see Table 1 for the $\mathrm{pH}$ before extubation for the overall population stratified by outcome, independently from cough assessment, indicating that there was a nonsignificant difference for $\mathrm{pH}$ ). There was no statistical 


\section{Predicting Extubation With Ventilator-Measured CPF}

Table 1. Characteristics of Early Extubation Success and Failure Groups in the 92 Subjects With Late Cough Peak Flow and Late Tidal Volume Measurements Available

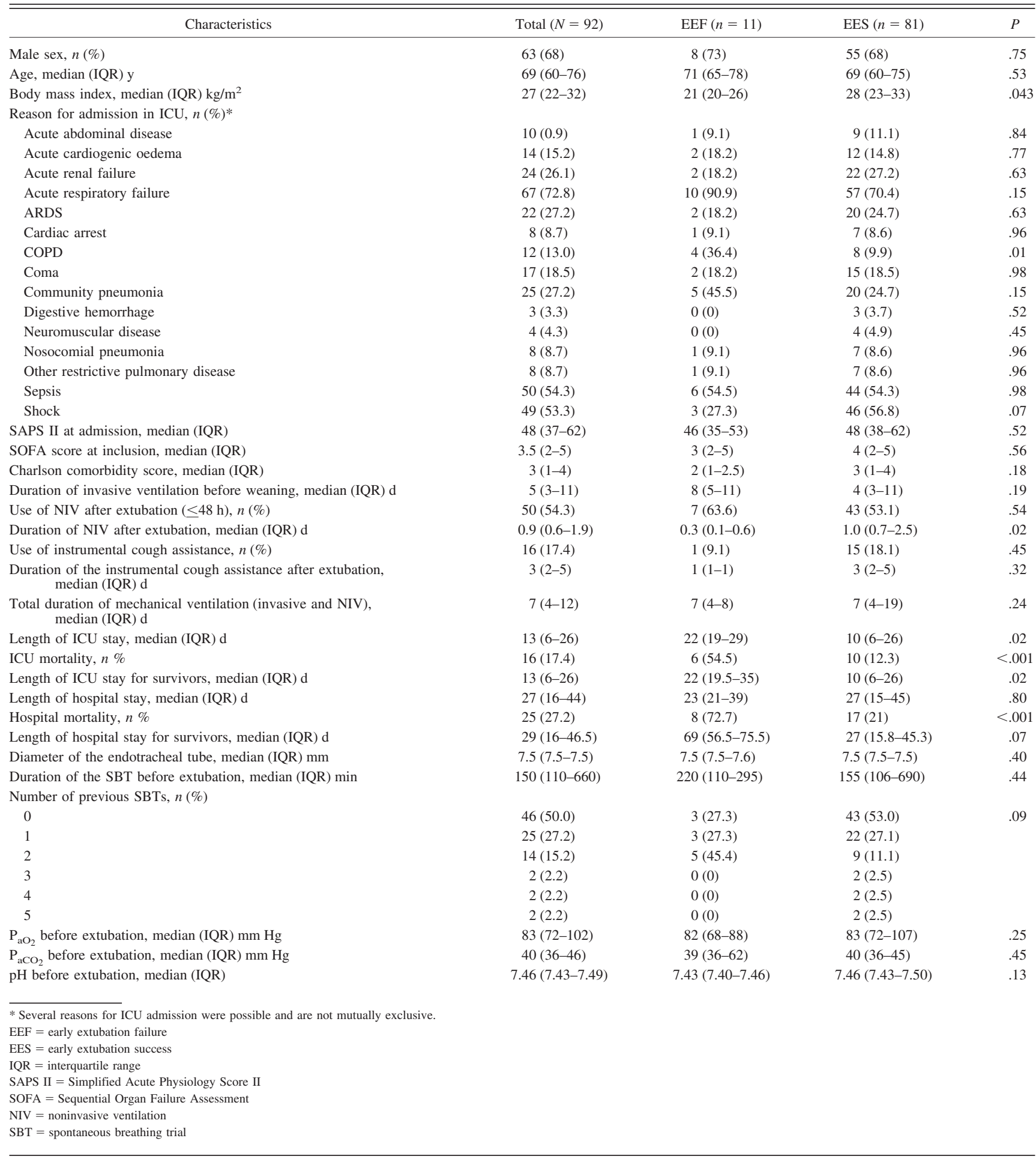

correlation between late $\mathrm{V}_{\mathrm{T}}$ and $\mathrm{pH}$ at the end of the SBT $\left(\mathrm{R}^{2}=-0.011, P=.91\right)$.

The extubation prediction score was thus built with $\mathrm{pH}$ alone, at a threshold of 7.45. The following combinations defined the risk of early extubation success: $\mathrm{CPF}<-60 \mathrm{~L} / \mathrm{min}$ or $\mathrm{V}_{\mathrm{T}}>0.55 \mathrm{~L}$ or $\mathrm{pH}>7.45$; the risk of early extubation failure: $\mathrm{CPF}>-60 \mathrm{~L} / \mathrm{min}$ and $\mathrm{V}_{\mathrm{T}}<0.55 \mathrm{~L}$ and $\mathrm{pH}<7.45$. Performance of the extubation prediction score was in- 


\section{Predicting Extubation With Ventilator-Measured CPF}

Table 2. Cause of Early Extubation Failure Along With Baseline Clinical Features in Subjects With High Cough Peak Flow and Low Cough Peak Flow

\begin{tabular}{cclcccl}
\hline \hline Subject & Age (y) & Sex & $\begin{array}{c}\text { SAPS II Score at } \\
\text { Admission }\end{array}$ & $\begin{array}{c}\text { SOFA Score at } \\
\text { Inclusion }\end{array}$ & CPF $<-60$ L/min & \multicolumn{1}{c}{ Extubation Failure Causes } \\
\hline 1 & 45 & Male & 38 & 5 & Yes & Hypoxemia \\
2 & 68 & Male & 30 & 2 & Yes & Hypoxemia \\
3 & 62 & Female & 49 & 2 & Yes & Surgery without quick extubation \\
4 & 72 & Female & 45 & 3 & Yes & Pulmonary edema or bronchial overload \\
5 & 69 & Female & 50 & 2 & No & Pulmonary edema \\
6 & 74 & Male & 48 & 5 & No & Hypoxemia and acute respiratory failure \\
7 & 62 & Male & 46 & 6 & No & Bulmonary edema, vomiting, and inhalation \\
8 & 78 & Female & 36 & 2 & No & Bronchial overload and acidosis \\
9 & 53 & Male & 37 & 5 & No & Hypercapnic acidosis \\
10 & 57 & Male & 62 & 6 & No & Acute respiratory failure and early hypercapnia \\
11 & 28 & Female & 27 & 2 & &
\end{tabular}

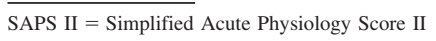

SOFA $=$ Sequential Organ Failure Assessment

$\mathrm{CPF}=$ cough peak flow

$\mathrm{NIV}=$ noninvasive ventilation

creased compared with the composite cough strength score (sensitivity $=96.3 \%$, 95\% CI 92.1-100; specificity $=54.5 \%, 95 \%$ CI 25.1-84; positive predictive value $=93.9 \%$, 95\% CI 88.7-99.2; negative predictive value $=66.7 \%, 95 \%$ CI 38.8-94.5).

\section{Performance of CPF and $V_{T}$ for the Secondary End Points}

Before Extubation to Predict Overall Extubation Success and Mortality. The performance of the composite cough strength score assessment (but not CPF alone; Table 3 ) to predict overall extubation success was also statistically significant $(P=.01$, positive predictive value $=83.3 \%, 95 \%$ CI 75.2-91.4). This composite criterion was not associated with ICU mortality but was associated with hospital mortality $(P=.042$, positive predictive value $=77.4 \%, 95 \%$ CI 68.7-86.8). Area under the curve values for both late CPF and late $\mathrm{V}_{\mathrm{T}}$ for each secondary end point were below those for the primary end point (Table 3).

\section{Assessment of CPF and $V_{T}$ Immediately After the SBT} Onset to Predict SBT Outcome. Early CPF and early $\mathrm{V}_{\mathrm{T}}$ were higher in case of successful SBT (Table 3). Using the same cut-off values for early CPF alone (CPF $<-60 \mathrm{~L} / \mathrm{min})$, no relationship was found with SBT success. In the absence of specific arguments in previous studies, cut-off values were selected from observed median values $\left(\mathrm{CPF}>-65 \mathrm{~L} / \mathrm{min}\right.$ and early $\left.\mathrm{V}_{\mathrm{T}}>0.55 \mathrm{~L}\right)$. No significant association was found between early CPF and SBT success $(P=.14)$, and this had low performance (sensitivity $=53.6 \%$, specificity $=66.7 \%$ ). Positive predictive value $(83.3 \%)$ was higher than negative predictive value $(31.5 \%)$ but was lower than the performance of late CPF to predict early extubation success.

\section{Discussion}

The novelty of this approach comes from the measurement of CPF by using the built-in ventilator flow meter without disconnecting the patient from the ventilator to estimate cough performance. The use of such a device without the need of a dedicated spirometer should increase the rate of routine assessment of cough performance and help clinicians in the decision making process at this stage. In this study, we measured experimentally the expiratory flow expelled from different inflationary lung volumes through an ETT alone and through an ETT attached to the expiratory circuit and valve of the ventilator used in this ICU. Cough performance assessment was also introduced to the clinical setting to define cut-off values predicting extubation outcome. Because our SBT is done at a lowpressure support level, we also assessed the volume at the end of the inspiration immediately preceding the cough as another indication of the respiratory system's ability to tolerate the extubation in increasing cough performance.

The first important finding of the present study was that CPF could be assessed with the built-in flow meter of an ICU ventilator, as demonstrated experimentally and confirmed clinically. Indeed, this is the first study measuring CPF using the flow meter of an ICU ventilator and demonstrating that it was as accurate to predict early extubation success as previously reported with a dedicated spirometer, $, 9,20$ and it is of note that the threshold was similar. More important, however, is the finding that early extu- 

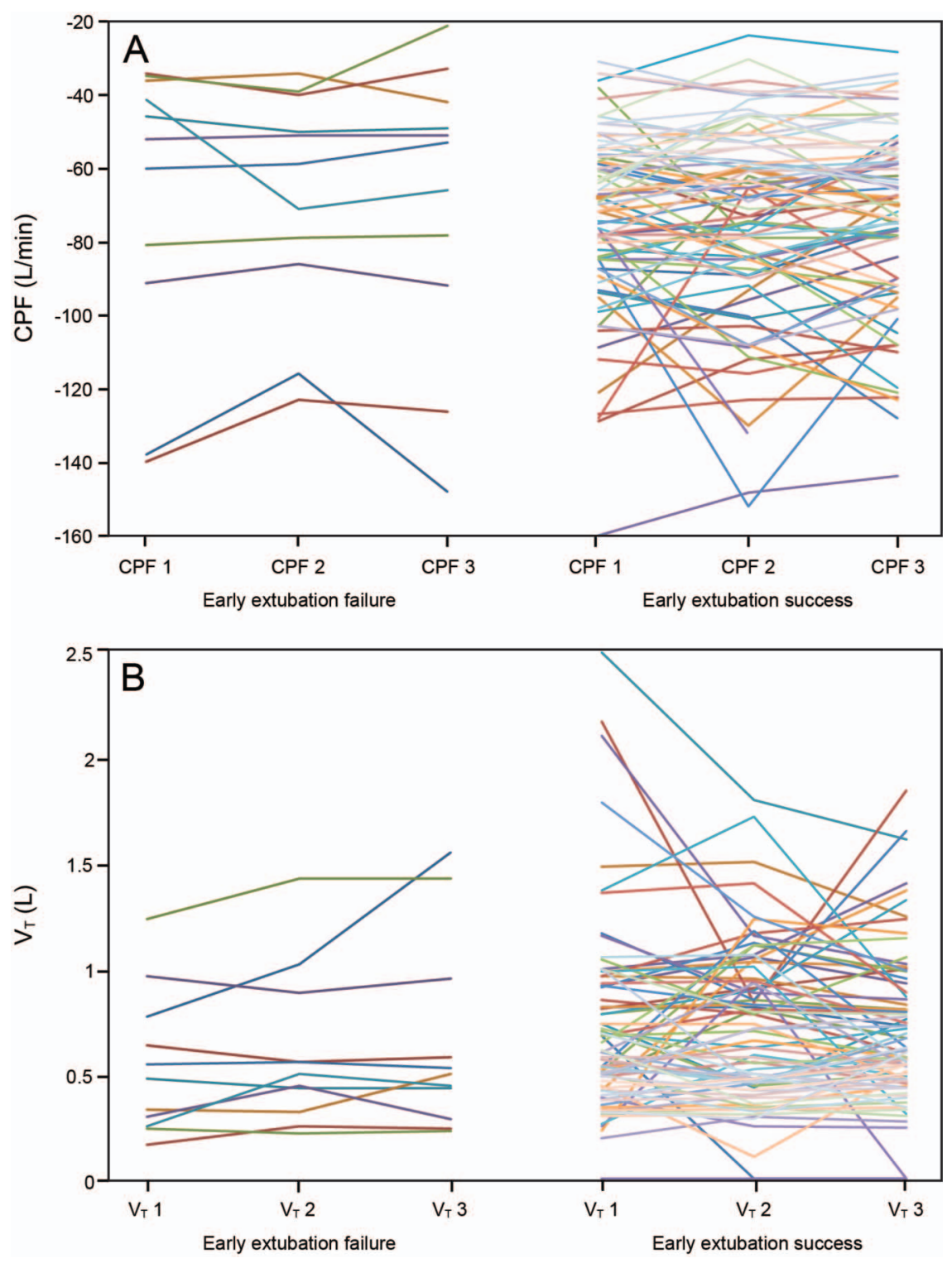

Fig. 5. Plot of individual variations of triplicate assessments. A: Between the first, second, and third assessment for late CPF, separated between early extubation failure and early extubation success, illustrating the absence of a significant trend with time for most subjects. B: Between the first, second, and third assessment for late $V_{T}$, separated between early extubation failure and early extubation success, illustrating the absence of a significant trend with time for most subjects.

bation success is more accurately predicted by a synergic analysis of several parameters, including $\mathrm{CPF}, \mathrm{V}_{\mathrm{T}}$, and $\mathrm{pH}$ before extubation.

CPF assessment has never been recommended in guidelines ${ }^{6,14}$ and was not an identified target of improvement in the quality of care in protocols. ${ }^{21}$ However, objective assessment has been called for ${ }^{13,22}$ because some publications assessing the clinical consequences of extubation failure were usually limited by the absence of objective assessment of cough strength. ${ }^{4}$ Furthermore, when objective assessments were employed, a variety of measurement conditions were used (spontaneous and voluntary cough ${ }^{15}$; spontaneous but involuntary cough ${ }^{10}$; manually assisted cough $^{12}$; and inclusion or not of neurosurgical subjects with oral endotracheal tubes or tracheostomy ${ }^{23,24}$ with possible severe cognitive impairments preventing an adequate response to cough instruction). This has also led to a wide range of cut-off values $(-80 \mathrm{~L} / \mathrm{min}$ was most adapted for reflex cough in the specific context of neuro-ICU ${ }^{24}$; $-35 \mathrm{~L} / \mathrm{min}$ was proposed in a medical ICU ${ }^{15} ;-29 \mathrm{~L} / \mathrm{min}$ was proposed for decannulation ${ }^{23}$ ). However, in the medical ICU context, a CPF threshold of around $-60 \mathrm{~L} / \mathrm{min}$ was the most consensual; the intrinsic performance initially observed for a threshold of $<-60 \mathrm{~L} / \mathrm{min}$ (sensitivity 

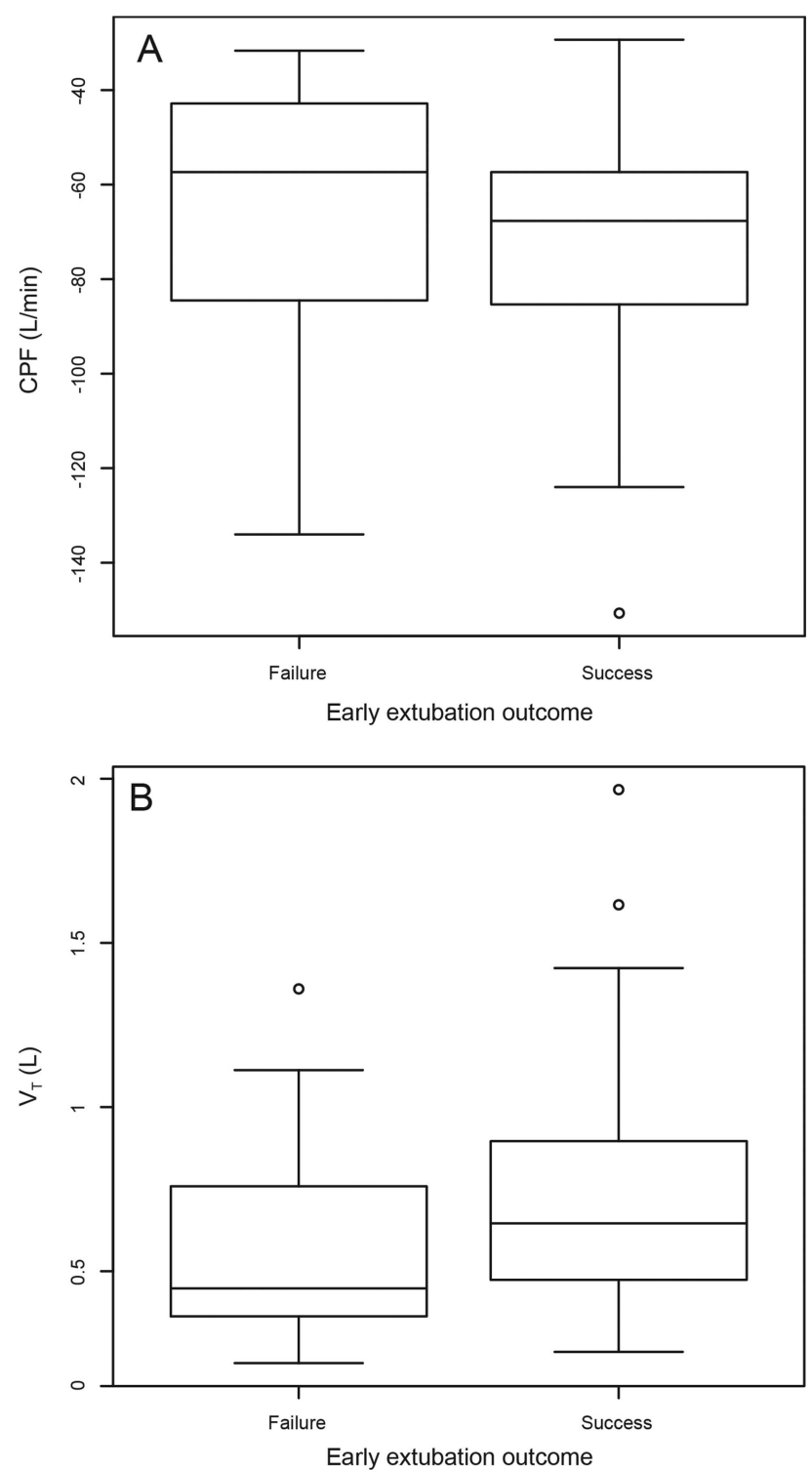

Fig. 6. Comparison of physiological results before extubation according to extubation outcome. Shown are box plots of late CPF (A) and late $V_{T}(B)$ in subjects who succeeded and in those who failed a scheduled extubation. The center line shows median value, the box denotes interquartile range between the first and third quartile, whiskers represent 1.5 times the interquartile range above the upper quartile and below the lower quartile, and dots represent outliers.

$69.0 \%$, specificity $74.0 \%$, relative risk 5.1 , area under the curve $=0.7)^{9}$ was later confirmed with cut-off values of $-58.5 \mathrm{~L} / \mathrm{min}$ (sensitivity $=71.4 \%$, specificity $=68.0 \%$, positive predictive value $=0.16$, negative predictive value $=0.94)^{11}$ and $-62.4 \mathrm{~L} / \mathrm{min}$ (sensitivity $=85.0 \%$, specificity $=64.2 \%$, area under the curve $=0.74) .{ }^{8,10}$ This objectively assessed threshold has been retained in a review $^{25}$ and was then used to select subjects for further interventional studies. ${ }^{20}$ The present study confirms pre-

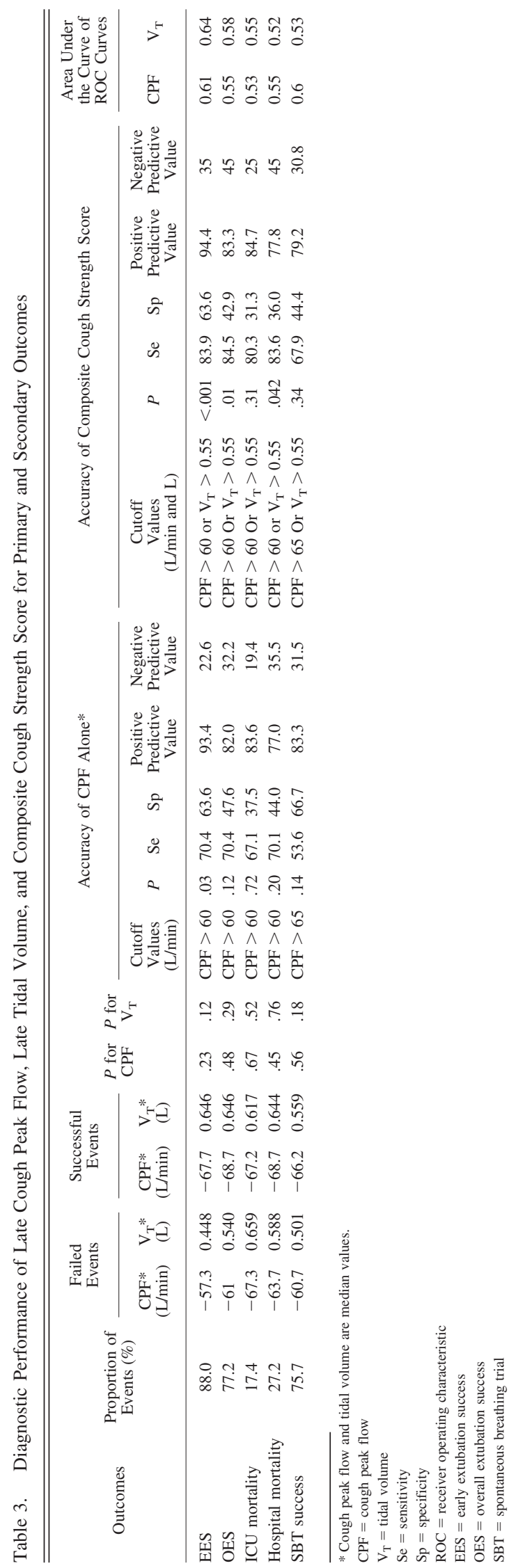



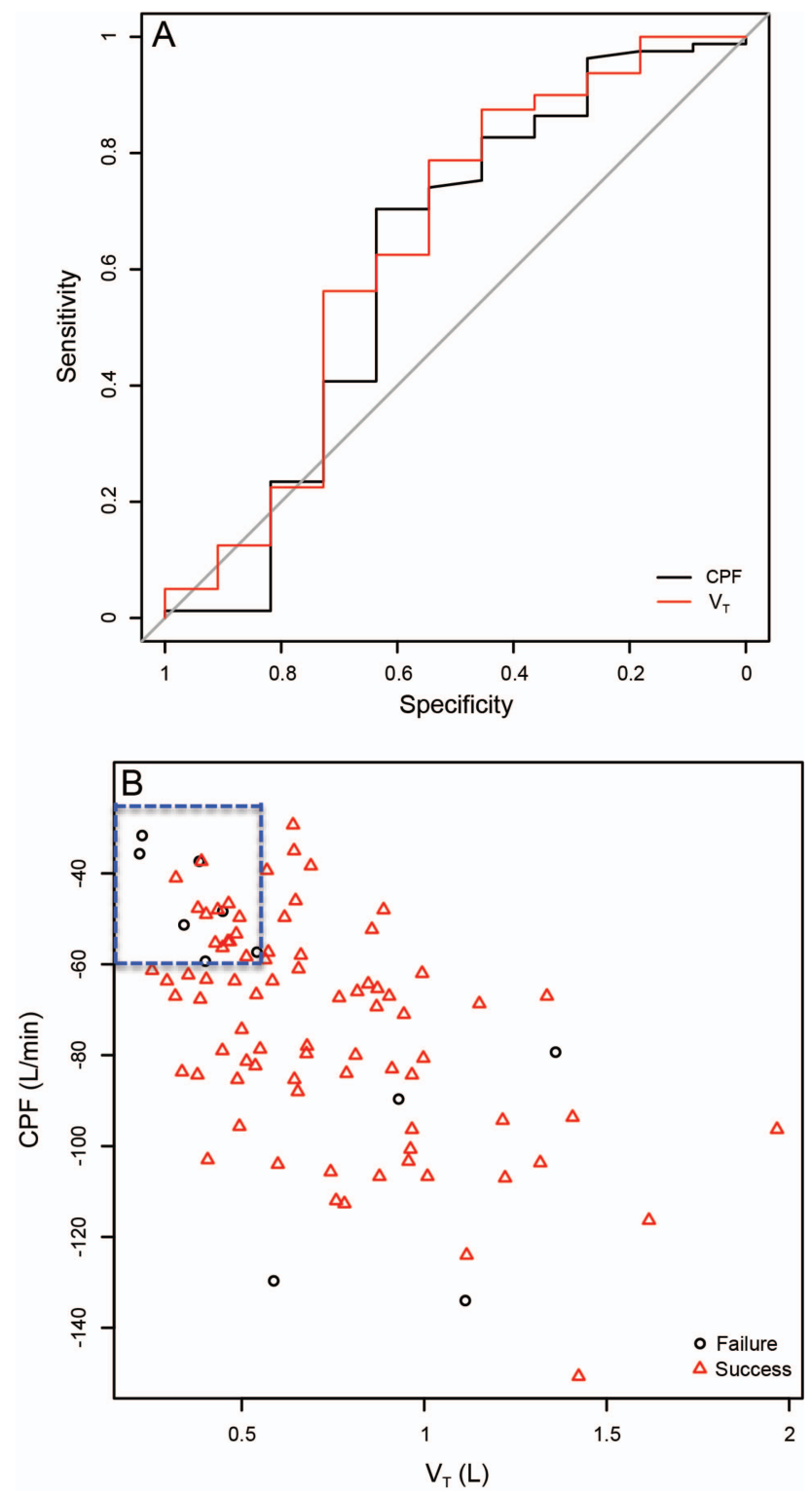

Fig. 7. A: Receiver operating characteristic curves and area under the curve values for late $\mathrm{CPF}(0.61,95 \% \mathrm{Cl} 0.37-0.83)$ and late $\mathrm{V}_{\mathrm{T}}$ (0.64, 95\% $\mathrm{Cl} 0.42-0.86)$ regarding early extubation outcome. $\mathrm{B}$ : Bivariate distribution of subjects according to late CPF and late $V_{T}$ for a visual validation of the thresholds previously defined (late $\mathrm{CPF}>-60 \mathrm{~L} / \mathrm{min}$ and late $\mathrm{V}_{\mathrm{T}}<0.55 \mathrm{~L}$ ). The population presenting a reduced cough strength according to the composite cough strength score is defined by a blue square, defining a cluster of early extubation failure cases (negative predictive value $=35.0 \%$ among this group). The population presenting a high cough performance (defined by 1 or 2 parameters above the threshold) had a high probability of early extubation success (positive predictive value $=94.4 \%$ ).

vious results regarding the accuracy of $\mathrm{CPF}$ assessment and may lead to an increased use in routine care.

The lack of homogeneity in the literature regarding the primary end point (eg, late decannulation success after 2 weeks ${ }^{12}$; early extubation success after $72 \mathrm{~h}^{9,22}$ ) led us to test the timing of the cough measure ${ }^{12}$ (before SBT or extubation) as secondary end points. According to the present study, the absence of weaning prediction (SBT outcome) by early CPF and early $V_{T}$ suggests that cough performance is specific to extubation outcome when measured before extubation (late $\mathrm{CPF}$ and late $\mathrm{V}_{\mathrm{T}}$ ). Another interesting point to note is that $\mathrm{V}_{\mathrm{T}}$ before cough has been previously reported with a comparable result: The higher the tidal volume, the greater the probability of extubation success. ${ }^{10}$ Nevertheless, this feature was never combined with other criteria to predict extubation outcome as it was herein in the composite cough strength score, which was found to be more significantly correlated with early extubation success. It is also important to note that this measure is easily performed at the bedside and is also associated with long-term clinical outcomes (overall extubation success and hospital mortality). However, we found no association between ICU mortality and CPF alone, which is in accordance with certain studies, ${ }^{8,10,15}$ although a relationship between extubation failure and Sequential Organ Failure Assessment worsening 4,5 or between low CPF and mortality ${ }^{9}$ has been reported. These results may illustrate the need for composite measures, such as the composite cough strength score, but also that mortality is multidimensional and that prognostic scores should consider a wider variety of potentially informative variables.

The contribution of the $\mathrm{pH}$ value before extubation to early extubation success prediction seems to be in line with the general knowledge in respiratory physiology. The protective effect of a higher $\mathrm{pH}$ could be considered an indicator of (relative) hyperventilation during SBT with minimal inspiratory pressure support $\left(7 \mathrm{~cm} \mathrm{H}_{2} \mathrm{O}\right)$ that reflects ventilation capacity over a longer period of time than $\mathrm{V}_{\mathrm{T}}$ (which is measured for only one inspiration). Furthermore, the absence of correlation between $\mathrm{pH}$ and late $\mathrm{V}_{\mathrm{T}}$ indicates that both should be considered for early extubation success prediction. This has not been reported in previous studies: For example, higher $\mathrm{pH}$ was not correlated with outcome by Thille et al, ${ }^{4}$ but objective cough assessment was not considered in their study, and when studies that were focused on predicting extubation by cough did investigate $\mathrm{pH}$, they failed to perform an analysis in the subgroup with low cough strength..$^{8,10}$ Taken together, this may explain the absence of recommendations to take into account higher $\mathrm{pH}$ in extubation decisions ${ }^{5}$ (abnormally low $\mathrm{pH}$ only being used as an SBT failure criterion $\left.^{14}\right)$.

\section{Limitations of This Study}

The study has several limitations. It is of note that the use of NIV could reduce the pertinence of comparison with studies previously mentioned. Even if a longer duration of NIV in case of early extubation success was ob- 


\section{Predicting Extubation With Ventilator-Measured CPF}

served, this result is spurious because each subject in the early extubation failure group had, by definition, $<48 \mathrm{~h}$ of NIV. Most importantly, though, the use of NIV was the same in both groups, reducing the risk of bias. In a recent study ${ }^{26}$ based on a larger population (356 ICU subjects), the use of NIV was associated with a lower risk of reintubation only for subjects with a low cough strength (defined as under the median value of $70 \mathrm{~L} / \mathrm{min}$ ). This value was notably close to the median value of late $\mathrm{CPF}$ that we found $(67.17 \mathrm{~L} / \mathrm{min})$ but was not discriminate for extubation outcome.

The cough assessment was restricted to 2 dimensions $\left(\mathrm{CPF}\right.$ and $\mathrm{V}_{\mathrm{T}}$ ) because the study was merely a pragmatic one, aiming at direct application. However, it cannot be completely ruled out that several other markers (eg, the cough volume instead of the pre-cough inspiratory volume $)^{19}$ could also be predictive. A physiological study including fully cooperative ICU subjects could address this point further. In the same vein, the comparison between CPF assessed with a dedicated spirometer and that assessed with the built-in ventilator flow meter may have been interesting. However, it would have been redundant with the bench study result.

An aspect that was not investigated herein is the semiquantitative cough strength score (from 0 to 5) proposed to bypass the lack of practical application of CPF. It has been reported to be equivalent to $\mathrm{CPF}$ assessed with a dedicated flow meter to predict a reduced rate of early extubation failure within $72 \mathrm{~h}$. However, as CPF was measured at the same time as subjective cough assessment, this measurement was not blinded. ${ }^{8}$ More generally, the consequences for clinical application remain uncertain because the predictive value depends more on clinician experience than the results of the test itself and therefore requires frequent training sessions.

Another limitation is the single-center design because this affects the generalizability of the results, in particular because the study was conducted in a medical ICU dedicated to respiratory care. This also affected the sample size, which could explain the nonsignificant association between late CPF and overall extubation success/hospital mortality (contrary to a previous study) ${ }^{9}$ or between early CPF and SBT success. The lack of statistical significance for ROC results could also be related to this issue. However, these are secondary end points. Another point is that statistical correlation made by repeated non-parametric tests was not controlled for multiple comparisons.

The study results were also affected by the low early extubation failure rate in the cohort, which is a paradoxical limitation because it may have reduced the power of statistical analysis through the reduction in the number of events. In particular, the low number of subjects in the early extubation failure group may explain the wide CI observed for all assessments of specificity and negative predictive value and consequently area under the curve, in particular without a proper sample size calculation. This limitation is shared by other studies, ${ }^{9,15}$ because the closer one gets to the objective of $5-10 \%$ extubation failure, ${ }^{7}$ the more difficult it is to demonstrate the efficacy of a novel strategy to challenge high-quality standard care. However, the WIND study ${ }^{27}$ (the most recent large observational cohort in France to be published) found that the usual extubation failure rate was $18.4 \%$, confirming that the present cohort is an outlier in this regard. This could be explained by the inclusion criteria of the present study rather than a center effect, because those without CPF assessment before extubation could have had a higher extubation failure rate than those included, and therefore the extubation failure rate of the study ICU could be closer to that reported previously. A difference in the overall management in this ICU could explain why the area under the curve value for late CPF was lower than in previous studies $\left(0.68,{ }^{8} 0.70,{ }^{9}\right.$ and $\left.0.74^{10}\right)$ and was not significant; however, the statistical significance of area under the curve values using the ROC method was provided in only one study, ${ }^{10}$ and none of these used a built-in ventilator flow meter.

Another limitation is that nearly two thirds of intubated subjects were not included for the primary end point analysis. However, other than the subjects who were not included due to omission (who represent $<10 \%$ for the primary end point), the reasons for non-inclusion were in accordance with the protocol. It is also worth noting that this information has rarely been reported in previous studies $^{8-11,24}$; the only study that seems to have provided these data was an investigation of a non-ICU decannulation protocol for which $38.5 \%$ of subjects were not included. ${ }^{23}$

This study was not restricted to a particular group of subjects according to the complexity of their weaning process. However, although the population was not formally described according to the more recently published "separation attempt," 27 an indirect comparison can be made. Half of the population had a CPF assessment for the first separation attempt, and the median value of mechanical ventilation was $5 \mathrm{~d}$, indicating that at least half were part of the group 2 ( $<1$ week of weaning process). However, the third percentile value was $11 \mathrm{~d}$, indicating that at least a quarter of subjects would have been classified in group 3 (prolonged weaning process $>1$ week).

\section{Strengths of This Study}

Despite a certain number of limitations, the study also has many strengths, the main one being that the approach was comprehensive, by assessing 2 features $\left(\mathrm{CPF}\right.$ and $\mathrm{V}_{\mathrm{T}}$, rather than $\mathrm{CPF}$ alone) at 2 time points (early and late, instead of only before extubation), and included several secondary end points (including long-term extubation out- 


\section{Predicting Extubation With Ventilator-Measured CPF}

comes and mortality, rather than early extubation success alone).

Introducing $\mathrm{V}_{\mathrm{T}}$ to increase prognosis accuracy indicates that a single assessment cannot capture the complexity of the subject state before extubation, although both parameters are correlated. Consequently, combined scores (composite cough strength score associating $\mathrm{CPF}$ with $\mathrm{V}_{\mathrm{T}}$; extubation prediction score if $\mathrm{pH}$ before extubation is added) increased the predictive value for success and failure. The composite cough strength score reached statistical significance to predict overall extubation success and hospital mortality, and, for extubation prediction score, the absence of statistical correlation between late $\mathrm{V}_{\mathrm{T}}$ and $\mathrm{pH}$ at the end of the SBT rules out a confounding effect of an overall increased minute ventilation on both parameters. Furthermore, $\mathrm{CPF}$ remained related to extubation prediction rather than the prediction of SBT success. Most importantly, this study indicates that CPF can be translated into routine ICU practice using built-in flow meters with the cut-off value $\mathrm{CPF}<-60 \mathrm{~L} / \mathrm{min}$. It is also of note that, since each measure was made by physicians, physiotherapists, or nurses, $\mathrm{CPF}$ assessment is not investigator-centered. Thus, learning curves in each ICU should be favorable.

\section{Perspectives for Therapeutic Strategies}

The results of the present study could encourage a widespread use of CPF measurement, which could reinforce the clinical confidence in extubation decisions. This study has demonstrated the validity in routine practice of previous thresholds and increased the performance of cough assessment by aggregating several measurements into composite predictive scores. Thanks to the high prediction of success, one could identify patients able to be extubated without complication. Conversely, it seems unwise to postpone extubation in case of low cough ability due to the lower predictive value of extubation failure. However, because patients most at risk have to be extubated anyway, the identification of these patients allows specific strategies preventing re-intubation to be targeted, and this should be tested in randomized interventional studies. Given the low number of early extubation failures, these studies must also confirm in a multi-center approach, and with a sufficient number of re-intubations, that prediction of extubation outcome remains after stratification on re-intubations required by bronchial overload. Furthermore, such studies that implement systematic cough assessment should then confirm the causal relationship between cough strength and outcome. To date, this kind of relationship has only been suggested in the subgroup of COPD with an invasive intervention postextubation (systematic fibroscopy) in the case of low cough. ${ }^{20}$ It has also been indirectly explored by an open-labeled study suggesting that systematic NIV might be useful in the case of low cough strength. ${ }^{26}$ How- ever, such interventions based on cough assessment must be validated with rigorous methodology and in a general ICU population.

\section{Conclusions}

CPF measured using the flow meter of an ICU ventilator was able to predict extubation success and build a composite score to predict extubation failure. The results were close to those found in previous studies that used a dedicated flow meter. This could help to identify high-risk patients to prevent extubation failure.

\section{REFERENCES}

1. Mekontso Dessap A, Roche-Campo F, Kouatchet A, Tomicic V, Beduneau G, Sonneville R, et al. Natriuretic peptide-driven fluid management during ventilator weaning: a randomized controlled trial. Am J Respir Crit Care Med 2012;186(12):1256-1263.

2. Esteban A, Frutos-Vivar F, Ferguson ND, Arabi Y, Apezteguía C, González M, et al. Noninvasive positive-pressure ventilation for respiratory failure after extubation. N Engl J Med 2004;350(24):24522460.

3. Khamiees M, Raju P, DeGirolamo A, Amoateng-Adjepong Y, Manthous CA. Predictors of extubation outcome in patients who have successfully completed a spontaneous breathing trial. Chest 2001; 120(4):1262-1270.

4. Thille AW, Harrois A, Schortgen F, Brun-Buisson C, Brochard L. Outcomes of extubation failure in medical intensive care unit patients. Crit Care Med 2011;39(12):2612-2618.

5. Thille AW, Richard JC, Brochard L. The decision to extubate in the intensive care unit. Am J Respir Crit Care Med 2013;187(12):12941302.

6. MacIntyre NR. Evidence-based guidelines for weaning and discontinuing ventilatory support. Chest 2001;120(6):375S-395S.

7. Krinsley JS, Reddy PK, Iqbal A. What is the optimal rate of failed extubation? Crit Care 2012;16(1):111.

8. Duan J, Zhou L, Xiao M, Liu J, Yang X. Semiquantitative cough strength score for predicting reintubation after planned extubation. Am J Crit Care 2015;24(6):e86-e90.

9. Smina M, Salam A, Khamiees M, Gada P, Amoateng-Adjepong Y, Manthous CA. Cough peak flows and extubation outcomes. Chest 2003;124(1):262-268.

10. Duan J, Liu J, Xiao M, Yang X, Wu J, Zhou L. Voluntary is better than involuntary cough peak flow for predicting re-intubation after scheduled extubation in cooperative subjects. Respir Care 2014; 59(11):1643-1651

11. Gao XJ, Qin YZ. [A study of cough peak expiratory flow in predicting extubation outcome]. Zhongguo Wei Zhong Bing Ji Jiu Yi Xue 2009;21(7):390-393.

12. Bach JR, Saporito LR. Criteria for extubation and tracheostomy tube removal for patients with ventilatory failure. A different approach to weaning. Chest 1996;110(6):1566-1571.

13. Chiou M, Bach JR, Gonçalves MR, Vudayagiri L. Misconceptions in the assessment of cough peak flow measurements for extubation or decanulation protocols. Revista portuguesa de pneumologia 2015; 21(5):285-286.

14. Boles JM, Bion J, Connors A, Herridge M, Marsh B, Melot C, et al. Weaning from mechanical ventilation. Eur Respir J 2007;29(5):10331056. 


\section{Predicting Extubation With Ventilator-Measured CPF}

15. Beuret P, Roux C, Auclair A, Nourdine K, Kaaki M, Carton MJ. Interest of an objective evaluation of cough during weaning from mechanical ventilation. Intensive Care Med 2009;35(6):1090-1093.

16. Colpart JJ, Moskovtchenko JF, Finaz de Villaine J, Mercatello A. [Brain death: diagnosis, physiopathology and management]. Cah Anesthesiol 1987;35(4):291-304.

17. Ferrer M, Sellarés J, Valencia M, Carrillo A, Gonzalez G, Badia JR, et al. Non-invasive ventilation after extubation in hypercapnic patients with chronic respiratory disorders: randomised controlled trial. Lancet 2009;374(9695):1082-1088.

18. Smith JA, Aliverti A, Quaranta M, McGuinness K, Kelsall A, Earis $\mathrm{J}$, Calverley PM. Chest wall dynamics during voluntary and induced cough in healthy volunteers. J Physiol 2012;590(3):563-574.

19. Hegland KW, Troche MS, Davenport PW. Cough expired volume and airflow rates during sequential induced cough. Front Physiol 2013;4:167.

20. Liu X, Li Y, He W, Xu Y, Sang L. [The application of fibrobronchoscopy in extubation for patients suffering from acute exacerbation of chronic obstructive pulmonary disease with low cough peak expiratory flow]. Zhonghua Wei Zhong Bing Ji Jiu Yi Xue 2014; 26(12):855-859.

21. Zhu B, Li Z, Jiang L, Du B, Jiang Q, Wang M, et al. Effect of a quality improvement program on weaning from mechanical ventila- tion: a cluster randomized trial. Intensive Care Med 2015;41(10): 1781-1790.

22. Winck JC, LeBlanc C, Soto JL, Plano F. Reply to "Misconceptions in the assessment of cough peak flow measurements for extubation or decanulation protocols". Rev Port Pneumol 2015;21(5):286-287.

23. Chan LY, Jones AY, Chung RC, Hung KN. Peak flow rate during induced cough: a predictor of successful decannulation of a tracheotomy tube in neurosurgical patients. Am J Crit Care 2010;19(3): 278-284.

24. Kutchak FM, Debesaitys AM, Rieder Mde M, Meneguzzi C, Skueresky AS, Forgiarini Junior LA, Bianchin MM. Reflex cough PEF as a predictor of successful extubation in neurological patients. J Bras Pneumol 2015;41(4):358-364.

25. Winck JC, LeBlanc C, Soto JL, Plano F. The value of cough peak flow measurements in the assessment of extubation or decannulation readiness. Rev Port Pneumol 2015;21(2):94-98.

26. Duan J, Han X, Huang S, Bai L. Noninvasive ventilation for avoidance of reintubation in patients with various cough strength. Crit Care 2016;20(1):316

27. Béduneau G, Pham T, Schortgen F, Piquilloud L, Zogheib E, Jonas $\mathrm{M}$, et al. Epidemiology of weaning outcome according to a new definition: the WIND study. Am J Respir Crit Care Med 2017;195(6):772-783.

This article is approved for Continuing Respiratory Care Education credit. For information and to obtain your CRCE

(free to AARC members) visit

www.rcjournal.com 